\title{
COMUNIDADES DE APRENDIZAJE: SOCIEDAD DE LA INFORMACIÓN PARA TODOS (CAMBIOS SOCIALES Y ALGUNAS PROPUESTAS EDUCATIVAS)
}

\author{
Carmen Elboj \\ Mercé Espanya \\ Ramón Flecha \\ Francesc Imbernon \\ Ignasi Puigdellívol \\ Rosa Valls \\ Universitat de Barcelona
}

\begin{abstract}
RESUMEN. En este artículo se exponen algunas propuestas para la educación dentro del marco de los cambios sociales (dualización social, revaloración de la educación, medios intelectuales...) que están teniendo lugar dentro de la sociedad de la información.
\end{abstract}

ABSTRACT.In this article, some tasks for education are pointed out in relation to the social changes (social dualization, education revaluation, intellectual means...) that are taking place within infomation society.

\section{Introducción}

Como el título indica, este artículo está dividido en dos partes. Por un lado, se explican las transformaciones sociales que están teniendo lugar en las sociedades europeas, las que afectan de formas diversas a las principales dimensiones de la vida cotidiana. Por el otro, desde la educación se señalan algunas tareas para superar los efectos perversos resultantes, como también alternativas para hacer frente a esta nueva situación. Concretamente, se formula una propuesta: las Comunidades de Aprendizaje como una vía diferente de proporcionar educación a aquellos grupos que están permanentemente excluidos. Aunque el ejemplo ha sido aplicado en escuelas de primaria se remarca su aplicabilidad a otros casos. 


\section{Cambios sociales en Europa}

\section{De la sociedad industrial a la informacional}

En un proceso de rápido avance, un nuevo tipo de sociedąd, está emergiendo y generando nuevos tipos de desigualdades. Desde la crisis de la sociedad industrial, la nueva situación se ha llamado sociedad postindustrial ${ }^{1}$ y se ha descrito básicamente como la ruptura con el pasado. Esta dinámica no explica la realidad de las puertas del siglo XXI, como tampoco podemos explicar el siglo XIX como la sociedad postfeudal argumentando que no es la sociedad feudal del siglo XV.

El paso de la sociedad industrial a la informacional tiene consecuencias que han transformado el contexto de la actividad económica y la manera en que funcionan nuestras sociedades. En este marco, el Libro Blanco de la Educación considera que la sociedad europea está en transición hacia una nueva forma de sociedad en la que es necesario responder a la necesidad de un amplio conocimiento de base y desarrollar habilidades para la ocupación y la vida económica. Todas estas nuevas condiciones el contexto tecnológico global y el advenimiento de la sociedad informacional-provocan nuevas necesidades para posibilitar el acceso a la información y al conocimiento.

En los últimos años, se han desarrollado importantes análisis prospectivos de la nueva realidad para entender la crisis e intentar dar soluciones al problema. Esto nos permite explicar la actual sociedad de la información de forma positiva, no sólo en función de las características que antes tenía la sociedad industrial sino también a partir de los elementos propios que definen el nuevo fenómeno. Estas contribuciones nos permiten determinar proyectos futuros para participar en la transformación.

Las tecnologías de la información han cambiado radicalmente la naturaleza del trabajo y la organización de la producción. Estos cambios están afectando profundamente a la sociedad europea. Las relaciones de producción y las condiciones de trabajo están cambiando. Las características de las tareas a desarrollar en el mundo laboral dependerán cada vez más de requerimientos intelectuales y de la habilidad de adaptarse. Por esta razón, las personas sin estas habilidades específicas están siendo excluidas del mercado laboral. En este sentido, la educación y la formación son uno de los factores determinantes en la igualdad de oportunidades.

La selección y el procesamiento de la información se convierten en un factor clave de la economía y de las otras áreas de nuestra vida social. El impacto de la revolución de la información es más acelerado y no menos trascendental que el que tuvo la industrial. Las características de la revolución informacional van desde el aumento de la importancia de las funciones del diseño (la información añadida al producto) al crecimiento relativo del poder del capital cultural de las familias, en lugar del económico, que determina el mayor o menor éxito de la formación.

Estas nuevas necesidades conllevan el acercamiento del sistema educativo y el productivo. Es también remarcable la convergencia entre las demandas del mercado

1. Le han Ilamado con otros nombres anteponiéndole el prefijo post: Amitai Etzioni usó el término postmodernismo. Ralf Dahrendorf habló de la sociedad postcapitalista. Otros la han llamado sociedad postburgesa, posteconómica, postsocialista, postbienestar, postcivilizada. 
laboral y la oferta de formación. En este sentido, como resultado de la aplicación de las nuevas tecnologías a los procesos productivos, la diseminación del conocimiento científico y tecnológico se necesita y se acelera. Los centros educativos y las empresas tienen que desarrollar un proceso de cooperación.

Este panorama implica que los procesos mentales sean mucho más decisivos que en la sociedad industrial. Cada vez más, lo que determina el éxito en las diferentes áreas de la vida social depende más de las características culturales de cada persona, del conocimiento y de las competencias que se poseen.

En el contexto de la sociedad de la información, el progreso tecnológico modifica las calificaciones profesionales que los trabajadores necesitan para tener acceso a nuevos trabajos o tareas. Así vemos como el mercado laboral prioriza más las competencias específicas individuales con las que la gente demuestra lo que sabe y como lo sabe, que la posesión de un título concreto. La educación y la formación contribuyen a la transformación social y personal, entendiendo la educación como un proceso de cambios permanentes a lo largo de toda la vida.

\section{Dualización de la sociedad}

Aunque la revolución de la información ha aportado una mejora de las condiciones de vida de la humanidad, el modelo social hegemónico que implica provoca una relativa agudización de las viejas desigualdades y genera otras nuevas. Entre ellas, aparece una división de los trabajadores como consecuencia de no utilizar las nuevas posibilidades para reducir la jornada laboral, sino para disminuir el número de puestos de trabajo que disponen de cierta estabilidad y remuneración. En este sentido, estamos alcanzando una clara división de los trabajadores en tres sectores: fijos, temporales y desempleados.

De acuerdo con la UE, hay un porcentaje del $60^{\prime} 4 \%$ de población activa que tiene ocupación. La organización tradicional del trabajo - basada en una jornada completa, ocupaciones muy definidas y un modelo de carrera profesional para toda la vida está cambiando. La situación de precariedad laboral es especialmente importante en España donde un 33'6\% de personas empleadas no tiene trabajo fijo. Este porcentaje es muy elevado si lo comparamos con el de otros países: Luxemburgo con un $2^{\prime} 6 \%$, Bélgica con un $5^{\prime} 9 \%$, Reino Unido con un $6^{\prime} 9 \%$, Italia con un $7^{\prime} 5 \%$ y Austria con un $8 \%$.

Observamos una tendencia hacía la flexibilidad/movilidad en la transformación del mercado laboral que se refleja en el crecimiento del trabajo a tiempo parcial y temporal. Estas tendencias son remarcables en Dinamarca donde el 15,7\% de las personas con empleo (75\%) están contratadas a tiempo parcial, en Holanda el $24^{\prime} 7 \%$ de $65^{\prime} 1 \%$, en el Reino Unido el $16^{\prime} 3 \%$ de $69^{\prime} 8 \%$ y en Suecia el $16^{\prime} 2 \%$ de $70^{\prime} 3 \%$. Los trabajos temporales se incrementan en aquellos países dónde el número de empleos a tiempo parcial es menor. En España el 33'6\% son empleos temporales y el $3^{\prime} 6 \%$ son empleos a tiempo parcial, en Grecia el $11 \%$ y el $2^{\prime} 6 \%$ y en Portugal el $10^{\prime} 4 \%$ y el $4{ }^{\prime} 4 \%$. Los trabajadores a tiempo parcial o temporales proporcionan la flexibilidad necesaria al mercado laboral.

En este contexto social, un sector privilegiado tiene una relación ventajosa con el mercado laboral (trabajos fijos y bien remunerados). Otro sector tiene una relación 
esporádica con el mercado laboral. Finalmente, están aquellas personas que nunca han tenido acceso al trabajo. Esta fragmentación social provoca una situación en que los empleados trabajan más cada día, mientras que los desempleados tienen cada vez menos oportunidades para obtener un trabajo.

En este sentido, el desempleo es un problema manifiesto en la UE. En España, el $22^{\prime} 1 \%$ de la población activa está desempleada, seguida de Finlandia con el $15^{\prime} 7 \%$, Francia con el $12 \% 4 \%$ e italia con el $12 \%$. Las mejores condiciones laborales se dan en Luxemburgo con el $3^{\prime} 3 \%$, Austria con el $4^{\prime} 4 \%$ y Holanda con el 6'3\%.

El problema del desempleo afecta especialmente a los jóvenes. Un $21^{\prime} 8 \%$ de los europeos entre 15 y 24 años de edad están desempleados. Destacan por encima de la media: España $(41 \%)$, Finlandia $\left(35^{\prime} 3 \%\right)$, Grecia (31\%), Francia $\left(28^{\prime} 9 \%\right)$ y Bélgica (33'9\%).

También es remarcable el $48 \%$ de europeos que son desempleados de larga duración. En algunos países, el porcentaje de personas que hace más de un año que están desempleados está aumentando. En Italia, hay un 65\% de desempleados de larga duración, en Bélgica un $61 \%$, en Irlanda un 59\%, en Grecia un 56\%, en España un $53 \%$ y en Portugal un $50 \%$.

Algunos defensores del modelo de la reestructuración social defienden que ocurre lo mismo que en la revolución industrial. En un momento inicial, supuso un empeoramiento de las condiciones de vida de grandes sectores de la población, pero a largo plazo contribuyó a su mejora. Desde una perspectiva crítica, podemos decir que dicha mejora no es una consecuencia automática derivada de una línea de progreso industrial, sino de la presión de los movimientos sociales como, por ejemplo, la lucha de los sindicatos por la jornada laboral de ocho horas. En todo caso, independientemente de la validez científica y ética que esta posición merezca, es evidente que la educación tiene que hacer alguna cosa para superar estas desigualdades.

La priorización de los recursos intelectuales de la sociedad de la información incrementa la importancia de los elementos curriculares. Esto conlleva, desde determinados modelos, que la educación y la formación supongan cada vez más un importante criterio de discriminación para pertenecer a uno de los tres sectores. Pero en una sociedad desigual no tenemos todos las mismas posibilidades de acceder a dichas habilidades. En este sentido, los desarrollos culturales tienden a convertirse en uno de los factores que configuran la dualización social (Habermas 1988/1983: 36), es decir, la selección natural del más fuerte.

\section{Europa multicultural}

Otra de las transformaciones remarcables acaecidas en el contexto de la sociedad de la información es el nuevo fenómeno de la inmigración. La cantidad de inmigrantes ha aumentado tres veces su número inicial; en este sentido, en 1950 sólo el 1'3\% de la población total era extranjera mientras que en 1990 un $4^{\prime} 5 \%$ del total estaba registrada como tal (Castells 1997). Esto afecta las relaciones sociales de diversas maneras. Como se ha visto en la sección dedicada a la dualización social, dos tercios de la población activa trabaja y el resto (un tercio) no tiene ningún tipo de calificación en el mercado laboral. Es específicamente en este último tercio donde podemos 
encontrar la mayoría de los inmigrantes que sólo tienen acceso, en el mejor de los casos, al trabajo más precario y marginal.

También esta afluencia de población ha producido el surgimiento de actitudes racistas que responden agresivamente a los recién llegados. Es un reflejo del nuevo racismo europeo, los resultados de partidos xenófobos que quedaron en segundo lugar en las elecciones de algunos países (Austria, Bélgica Flamenca) y han crecido recientemente en otros (Noruega, Dinamarca).

\section{Clarificación de los conceptos}

Hay una gran dispersión de los términos en este asunto. La siguiente clasificación es sólo para esclarecer su uso en este artículo. Hay una pretensión de universalidad porqué muchos autores utilizan los mismos términos con distintos significados. El multiculturalismo se entiende como el reconocimiento de la existencia de diferentes culturas en un mismo territorio. El interculturalismo es una manera de intervenir en esa realidad que enfatiza la relación entre culturas. El pluriculturalismo es otra forma de intervención que pone el acento en la preservación de las diferentes identidades culturales.

Desde una perspectiva intercultural, es más positiva la coexistencia en una misma escuela de personas pertenecientes a diferentes culturas, ya que aquellas que se basan únicamente en una cultura tienden a ser vistas como ghettos. El Pluriculturalismo da más valor a la posibilidad de crear escuelas basadas en las diversas culturas como instrumento que permita desarrollar la suya propia. Por ejemplo, la idea de una escuela gitana ${ }^{2}$ en Barcelona puede ser vista como un ghetto desde el interculturalismo y como una oportunidad para la identidad cultural desde el pluriculturalismo.

\section{Enfoque etnocéntrico : interculturalidad como asimilación o integración.}

La modernidad tradicional adopta un enfoque etnocéntrico. Establece una cultura dominante tanto porque se considera superior como porque se considera original y característica de una nación o territorio específico. Desde esta creencia hay una intención explícita o implícita de destrucción de otras culturas. Dicen estar en contra de la existencia de escuelas basadas en otras culturas e incluso de la introducción de algunos elementos en el curriculum para evitar abandonos. Sin embargo, su elitismo se esclarece cuando observamos que este ataque no se hace extensivo a la existencia de escuelas francesas o alemanas ni a la introducción de elementos anglosajones en las culturas latinas.

La versión más explícita y conservadora del etnocentrismo es la que parte de nuevas posturas racistas y xenófobas. La idea central del nuevo racismo consiste en que cada etnia y cultura debe permanecer en su "territorio originario". Para conseguir esto defienden mecanismos de exclusión institucional como la obstrucción a la entrada de

2. En este artículo, una escuela gitana se entiende como una escuela dónde la educación se base en la cultura gitana, aunque haya la contradicción entre la cultura gitana y los valores inherentes en las características occidentales de la escuela. Esto no significa una escuela donde gente no española no puede ser matriculada. 
inmigrantes procedentes de otro país y violentas exclusiones como son los maltratos físicos a aquellos inmigrantes que ya residian en el país.

Entre las opciones que, hasta cierto punto, aceptan el hecho multicultural, el interculturalismo emerge como una manera de conseguir la asimilación y la integración. Desde esta óptica se acepta a las personas procedentes de otras culturas y se les facilita la adquisición progresiva de la cultura dominante. Este enfoque etnocéntrico se autoconsidera no racista ya que cuando una persona ha de ser evaluada o seleccionada para un puesto de trabajo no se tiene en cuenta su origen o el color de su piel. Sin embargo desde este posicionamiento también se reproducen las desigualdades ya que, a menudo inconscientemente, las reglas de juego, que son las de la cultura dominante, están previamente definidas. En otras palabras, si el sistema educativo es una institución masculina, blanca y occidental, la mayoría de personas de otras culturas fracasaran y sólo unas pocas triunfaran pero a costa de renunciar a su propia identidad.

\section{El enfoque relativista: pluriculturalismo para preservar las identidades}

Los enfoques nietzschianos adoptan posiciones relativistas (Foucault 1992/197576). El enfoque relativista está basado en la oposición a los principios universales modernos de igualdad, libertad y fraternidad. La relación entre las culturas durante los dos últimos siglos en el marco de la modernidad es considerada como una dinámica de destrucción de las personas. Las instituciones de la Educación se consideran como una arma crucial en el proceso de exclusión y destrucción. Estas creencias argumentan la imposibilidad de un dialogo libre entre culturas diferentes y no autorizan ningún intento de intervención, evaluación o incluso de análisis cuando no está basado en una cierta cultura. En este sentido, la propuesta principal es preservar y desarrollar la identidad cultural propia.

Desde la clasificación de términos que adoptamos en este artículo, el pluriculturalismo es el único enfoque posible, teniendo en cuenta la consideración que cualquier acción intercultural se entiende como un etnocentrismo escondido porque no se pueden desarrollar dentro de las condiciones ideales de dialogo libre entre iguales. La propuesta de una educación pluricultural es una contradicción en este enfoque (más todavía en la educación intercultural) porqué la creación de los sistemas educativos y las escuelas son una práctica que proviene de tipos específicos de culturas.

El enfoque relativista, desde sus versiones coherentes, tiende a reproducir y a incrementar desigualdades, ya que no tiene en cuenta que el origen de las desigualdades no sólo depende del incremento de relaciones entre culturas durante la modernidad, sino también de las características de cada cultura. En todas las culturas, hay importantes desigualdades, en función de diferentes factores como pueden ser el género o la edad. No hay una superación de esas desigualdades preservando las identidades culturales, sino a partir de su evolución. El relativismo se opone radicalmente a este desarrollo.

Enfoque comunicativo: interculturalismo y pluriculturalismo como opciones de una dinámica de libre diálogo hacia la igualdad.

El enfoque comunicativo desarrolla una propuesta basada en el diálogo como forma de relación entre las culturas y como camino hacia la superación de las desi- 
gualdades y exclusiones actuales (Habermas 1989/1987-88; Habermas 1997). Considera que la modernidad cultural ha implicado la destrucción de algunas culturas, pero también la divulgación de ideas y prácticas contradictorias con las desigualdades como, por ejemplo, el principio del derecho de todas las personas a la alfabetización, independientemente de su etnia, edad o género.

La homogeneidad ha producido efectos excluyentes y la destrucción de la riqueza cultural. Por otro lado, cuando la igualdad se incluye como el respeto a la diferencia genera unos efectos igualitarios muy importantes que contribuyen a superar los terribles efectos excluyentes que ya existían en la premodernidad.

El enfoque comunicativo propone intentar, tanto como sea posible, involucrar a las personas en la toma de decisiones en todos aquellos procesos en los que están implicadas. Por esta razón, se considera que la simultaneidad de interculturalismo y pluriculturalismo ofrece mayor pluralidad de opciones y consecuentemente más libertad para más personas. La existencia de escuelas españolas en Nueva York da la posibilidad a mucha gente de escoger entre ir a una escuela española o a una escuela más intercultural, entre dar una prioridad a la preservación de la identidad propia o el acercamiento hacia la cultura predominante en su entorno social.

Pero el enfoque comunicativo es crítico con los contextos sociales y consigo mismo. Analiza como, en las condiciones sociales actuales, la cultura dominante continua estando presente en las propuestas educativas que se plantean. Sin embargo, considera que tanto el etnocentrismo como el relativismo preservan y refuerzan las presentes desigualdades. Por su parte, el diálogo no elimina las desigualdades pero tiende hacia su eliminación.

La propuesta consiste en reducir los efectos exclusores e incrementar los igualitarios en la relación entre culturas a través del diálogo. Esto implica la ruptura de la homogeneidad cultural, haciendo permeables las diferentes culturas, incluida la cultura dominante. Esto comporta el enriquecimiento mutuo a través de procesos de mestizaje cultural.

\section{Diversidad e igualdad en educación}

\section{Homogeneidad desigual contra la diversidad}

Los nuevos derechos culturales lanzan una homogeneización ofensiva centrada en la Cultura en mayúsculas, es decir en su concepto más limitado y tradicional. Desde este punto de vista, factores tan diferentes como el relativismo cultural, la renovación pedagógica, la deficiente formación del personal, la democratización de las escuelas, la discriminación positiva son la causa de una pérdida de nivel por parte del sistema educativo. Esta ofensiva es una nueva forma de la imposición de la modernidad tradicional de la cultura masculina, blanca y occidental excluyendo al resto de identidades y culturas.

Autores como Bloom (1987) y Hirsch (1987) han destacado en Estados Unidos como los intelectuales líderes de esta ofensiva. Rorty (1989) cree que es necesario distinguir entre las dudas de Bloom sobre democracia y los deseos de Hirsch de un electorado democrático más educado. Disiente de la idea de Bloom de que la educación 
superior tiene que servir para que los estudiantes alcancen la superioridad natural de aquellos que son líderes de la vida teórica. De todos modos, está de acuerdo con Hirsch en que en los Estados Unidos no se ofrece una educación secundaria que permita a los niños actuar como ciudadanos en una democracia.

Sin embargo, los dos defienden una cultura sexista, etnocéntrica, clasista y edista. Los dos marcan un limite entre la cultura y la no-cultura, entendiendo la cultura del hombre blanco occidental de clase alta-media y con un nivel académico alto adquirido en la preadultez.

El caso de Bloom es claro porque su propuesta se basa en la excelencia de la élite evitando que su formación se obstruya con las tendencias democráticas que los contaminan con la cultura y la gente popular. Hirsh reclama una cultura homogénea para todo el mundo, pero parte de la cultura dominante, marginando a aquellos que no pertenecen a la cultura blanca, masculina y de clase alta o media.

\section{Diversidad como alternativa a la igualdad}

La diversidad, la diferencia o propuestas similares son el punto de partida de muchas reformas curriculares orientadas a la definición de diferentes itinerarios curriculares para adaptarse a las características de colectivos y estudiantes. Esta opción ha sido defendida como forma de respetar la diversidad de culturas, contextos y personas legitimada a través de argumentos relativistas. Pero estas perspectivas obvian el hecho de que todas las sociedades donde estas reformas tienen lugar no son sólo diversas sino también desiguales. El respeto a la diversidad basado en la mera adaptación a las diferentes realidades sociales y personales esconde en realidad una adaptación a las desigualdades.

En una sociedad desigual la tendencia predominante es que los diferentes itinerarios curriculares reproduzcan las desigualdades educativas previamente existentes. Una extensa serie de factores (posibilidades económicas, expectativas contextuales, cultura familiar, arbitrariedad cultural de las escuelas...) obliga a los grupos a adaptarse a criterios como clase social, género, etnicidad o edad. La simple adaptación a las diferencias los refuerza ya que se limita a reproducir la cultura de origen y los factores de desigualdad como parte de ella.

Si el objetivo no consiste en alcanzar un nivel de aprendizaje homogéneo para todos, sino respetar los diferentes procesos, olvidando el contexto de desigualdad social, entonces los diferentes itinerarios curriculares comportan niveles de educación muy desiguales. Por ejemplo, mientras que las escuelas a las que asisten personas procedentes de los grupos privilegiados se centran mayoritariamente en el aprendizaje competitivo en los niveles superiores de las instituciones académicas y de la sociedad, muchas escuelas públicas en áreas pobres se centran en pasar el tiempo lo más pacíficamente posible e intentando hacer aprender algo a los niños.

Cuando esto sucede, estamos adaptando a los estudiantes, a una edad muy temprana, a los nuevos tipos de desigualdades resultantes de la dualización de la sociedad informacional. Desde el sector de los profesionales altamente cualificados, con una alta remuneración y reconocimiento social hasta los desempleados estructurales que viven en condición de exclusión social. Las habilidades más competitivas son requeridas por los niveles más altos de esta escala, mientras que los más bajos deberían aceptar habitualmente su subordinación. 
Esta actitud se centra en la adaptación al contexto y, en la práctica, a aquellas habilidades de los estudiantes que son considerados y tratados como iguales. Algunas veces estas actitudes adaptativas son legitimadas con teorías opuestas como la Teoría Transformativa de Vygotsky (1986). Este autor relaciona el contexto y el desarrollo cognitivo de forma que el segundo comporta transformaciones socioculturales. Pero desde algunas perspectivas adaptativas se obvia la perspectiva transformativa, proponiendo adaptar los diferentes desarrollos cognitivos a los contextos sociales existentes.

\section{Igualdad de diferencias: igualdad incluyendo la diversidad}

La igualdad es un objetivo más global que la diversidad, la diferencia o la opción. La igualdad incluye el derecho de todo el mundo para escoger ser diferente y ser educado en la propia diferencia. Cuando la diferencia no le da importancia a la igualdad es porque, consciente o inconscientemente, está más a favor de sus efectos exclusores que de los igualitarios. Cuando en nombre de la igualdad no se tiene en cuenta la diferencia, se impone un modelo homogéneo de cultura que produce exclusión y desigualdad.

El objetivo de la igualdad no se puede alcanzarse proponiendo que todas las personas hayan de adquirir los mismos conocimientos, ya que este planteamiento no sólo no sirve para superar las actuales desigualdades culturales y educativas sino que contribuye a la reproducción del conjunto de desigualdades. La cuestión no es que todo el mundo tenga las mismas oportunidades de tener una cultura homogénea, sino redistribuir los recursos humanos y materiales para que nadie quede excluido por su diferencia para acceder a determinados elementos culturales. Freire ha combinado estos dos elementos aparentemente contradictorios. Propone partir de la propia cultura, pero no quedarse en este punto, sino conseguir el dominio de la propia realidad cultural.

En las nuevas perspectivas críticas, esta redistribución necesita una movilización de los ricos recursos humanos y culturales de aquéllos que actualmente son excluidos porque se consideran deficientes. Pero la deficiencia no está en las personas excluidas sino en la escuela y en la sociedad que no sabe sacar provecho de la riqueza cultural de los diferentes grupos o personas. Las políticas compensatorias se basan en las teorías del déficit, las cuales crean expectativas negativas en el aprendizaje que se convierten en profecías que inexorablemente se cumplen. La consideración positiva de las capacidades de aprendizaje de los participantes y de los recursos básicos movilizados para desarrollarlos, forma parte de la lucha de los nuevos movimientos culturales.

La educación igualitaria es uno de los objetivos de los movimientos y personas que luchan por la igualdad social. Si este objetivo se limita a buscar la igualdad de oportunidades para acceder a posiciones desiguales en la sociedad, constituye otra forma de legitimación de las desigualdades. Si los movimientos educativos y los trabajadores culturales están en contacto con otros movimientos sociales, apartándose del corporativismo que intenta cerrar la escuela, contribuyen a la lucha contra las desigualdades educativas y sociales. 
En la nueva perspectiva crítica, necesitamos desarrollar tanto prácticas como teorías alternativas. Algunos de los mejores pedagogos (Freire:1997), psicólogos (Vygotsky:1986) y teóricos sociales (Habermas:1996/1992) han sido y son personas comprometidas con la transformación social. Frecuentemente, una experiencia o teoría se considera deficiente si es transformadora y de calidad si está a favor del poder. Los autores y las instituciones que lamentablemente establecen estos acientíficos paralelismos, buscando protección en un conocimiento técnico, utilizan la cultura y la educación como barreras para fomentar las desigualdades. Estas perspectivas conservadoras han servido para destruir ilusiones, provocar monotonía y desmovilizar a estudiantes, movimientos sociales y profesores.

Es hora de revelarse y reclamar el derecho a ser diferente de aquellos que no hacen nada contra las nuevas desigualdades. Necesitamos imaginar un mundo mejor, escuelas mejores, pero no para imponer esto de manera autoritaria, sino para tener ese punto de utopía que los principales autores de las ciencias sociales (Habermas 1988/1984; Giddens 1990) consideran esenciales en toda perspectiva progresista. Después de estos años de desorientación, podemos empezar a preparar desde la práctica y la teoría una nueva perspectiva crítica que Freire, en una comunicación en Barcelona tituló: "nuestras posibles utopías".

\section{Efecto Desnivelador}

\section{La antigua concepción compensatoria}

La investigación sobre el efecto desnivelador ha generado un cambio total en las teorías y las prácticas de la educación de personas adultas en Europa. En España, uno de los cambios más relevantes se hizo durante la elaboración de la reforma educativa de la actual ley. El capítulo XIV del Proyecto para la Reforma Educativa de 1987 aún se basaba en la falsa creencia de que la escolarización eliminaría o reduciría las deficiencias de educación básica de las futuras personas adultas. Se dice: La educación básica que no se ha terminado a una edad correcta, se convierte en un requerimiento compensatorio esencial (MEC 1987, p.32). Por tanto, identifica la educación básica de personas adultas con la propia de la escolarización obligatoria que no se tenía.

El antiguo concepto compensatorio se basaba en los cinco errores siguientes:

a) Producto del pasado: las personas adultas que necesitan educación básica son aquellas que no han ido a la escuela o que no han completado sus estudios.

b) Componente de subdesarrollo: los países que tienen el "problema" es porque todavía les falta un buen nivel de desarrollo.

c) La reforma como solución: los niños y las niñas actuales son las futuras personas adultas. La solución es extender y mejorar la educación inicial. En este sentido, la educación básica no será necesaria en el futuro.

d) Edad apropiada: las edades preadultas son las mejores para aprender. Durante la adultez las personas tienen dificultades y lo mejor es concentrar la educación a temprana edad. 
e) Paralelismo con el sistema escolar: el curriculum de la educación obligatoria se ha de adaptar a las necesidades de las personas adultas.

\section{La nueva concepción basada en las capacidades}

El efecto desnivelador se produce por la ampliación de los años de enseñanza obligatoria. La LOGSE (Ley Orgánica General del Sistema Educativo, 1990) ha establecido en dieciséis años la edad para poder obtener el título correspondiente a la escolarización obligatoria, mientras que la Ley General de Educación (LGE) fijaba dicha edad en los catorce años. Esta ampliación implica la aparición de un nuevo nivel educativo socialmente requerido. Por tanto, todas las personas que han estudiado de acuerdo con el anterior sistema educativo quedan fuera de la ley educativa.

Las personas jóvenes y adultas que tenían el nivel educativo obligatorio antes de la implantación de la LOGSE, ahora no lo tienen. La LOGSE ha aumentado y aumentará el número de personas que no han completado el nuevo nivel obligatorio establecido por la Ley. Estas personas no tendrán el nivel mínimo requerido para obtener un trabajo o para ser admitidos en ningún programa, quedando en situación de desventaja.

EL EFECTO DESNIVELADOR: CRECIMIENTO DE LA POBLACIÓN MAYOR DE 16 AÑOS SIN LA TITULACIÓN CORRESPONDIENTE AL NIVEL OBLIGATORIO EN ESPAÑA POR FRANJA DE EDAD

\begin{tabular}{|c|c|c|c|}
\hline EDAD & 1981 & 1991 & $\begin{array}{c}2000 \\
\text { (Previsión) }\end{array}$ \\
\hline $16-24$ & $\begin{array}{c}622.877 \\
(10.03 \%)\end{array}$ & $\begin{array}{c}3.472 .238 \\
(59.29 \%)\end{array}$ & $\begin{array}{c}0 \\
(0 \%)\end{array}$ \\
\hline $25-44$ & $\begin{array}{c}2.908 .418 \\
(31.29 \%)\end{array}$ & $\begin{array}{c}7.097 .680 \\
(65.54 \%)\end{array}$ & $\begin{array}{c}7.045 .757 \\
(58.71 \%)\end{array}$ \\
\hline $45-64$ & 4.430 .867 & $\begin{array}{c}7.436 .141 \\
(87.62 \%)\end{array}$ & $\begin{array}{c}7.491 .372 \\
(79.01 \%)\end{array}$ \\
\hline más de 65 & 2.877 .868 & 4.976 .463 & 5.449 .587 \\
$(67.92 \%)$ & $(9.840 .030$ & 22.982 .522 & $(91.21 \%)$ \\
\hline TOTAL & $(38.71 \%)$ & $(75.29 \%)$ & $\begin{array}{c}19.986 .716 \\
(61.58 \%)\end{array}$ \\
\hline
\end{tabular}

Fuente: Estadísticas elaboradas a partir del censo de la población española de 1981 (INE 1985) y 1991 (INE 1994) y a partir de las previsiones sobre población de Del Hoyo \& García (1988).

En este contexto, el aprendizaje a lo largo de toda la vida adquiere una importancia aún mayor. La aceptación de las consecuencias del efecto denivelador provoca un cambio total en la concepción de la Educación de personas Adultas. En este sentido, el Capítulo III de la LOGSE, dedicado a la Educación de personas Adultas, significa un paso adelante. El Capítulo XII del Libro Blanco de la Reforma, corrigió los errores de la concepción compensatoria, abriendo paso a un nuevo concepto basado en las 
capacidades: La Educación Básica requerida por las personas adultas debe adaptarse a la presente evolución de la sociedad (MEC 1989a: 193).

La nueva concepción basada en las capacidades se fundamenta en las siguientes ideas:

a) Producto de la evolución actual y futura: la necesidad de la educación básica no es consecuencia de una escolarización deficiente sino de la constante evolución de la sociedad. La aparición de la sociedad de la información (Cardoso: 1993) ha provocado un cambio en las necesidades educativas de las personas adultas, diferentes de las que necesitaban hace unos años (Castells: 1994). Paralelamente, es importante renovar constantemente los conocimientos, ya que aquellos que adquirimos en la educación inicial no sirven para toda la vida. El Título III recoge esta idea a través de la extensión de la educación básica de personas adultas (art. 52) y de la secundaria no obligatoria.

b) Componente esencial de desarrollo: cuando más desarrollado es un país, más se adapta la educación básica de personas adultas a las necesidades de éstas. Los países menos desarrollados, particularmente aquellos que tienen regímenes dictatoriales, se han caracterizado por la adopción de la concepción compensatoria de la educación de personas adultas. En estos países, la educación de personas adultas desempeña un papel marginal, ya que no es considerada un elemento importante para el desarrollo del conjunto de la sociedad.

Desde esta perspectiva, la educación básica de personas adultas tiene que estar integrada en una concepción amplia de la educación de personas adultas junto con la formación ocupacional y la educación cultural y para el ocio, idea recogida por la LOGSE en su artículo 51.5.

c) Las reformas educativas producen un efecto desnivelador: cada nueva reforma aumenta el nivel básico y obligatorio. Con la ampliación del nivel secundario obligatorio hasta los 16 años, las personas que habían obtenido el Graduado Escolar están ahora por debajo del nuevo nivel. El Capítulo III trata de superar el efecto desnivelador de la presente reforma y la exclusión cultural y social generada por la sociedad de la información.

d) Cualquier edad es adecuada para aprender: durante las tres últimas décadas la investigación sobre educación de personas adultas demuestra como la capacidad de aprender durante la adultez no es mejor ni peor que durante la infancia o la juventud, sino diferente. Los estudios sobre la inteligencia cristalizada (Cattel 1971) y, actualmente, sobre inteligencia práctica (Martin \& Scribner 1991, Scribner 1988) e inteligencia cultural (CREA 1997) son suficientes para afirmar que cualquier edad es buena para aprender.

e) Curriculum específico: La concepción de curriculum de la reforma da importancia a los estadios evolutivos. Uno de los objetivos básicos del nuevo curriculum (MEC 1989b:32) es adaptar la educación a los estadios evolutivos de la infancia y de la juventud. La educación de personas adultas no se tiene que adaptar a la educación infantil y juvenil, sino que se ha de basar en los estadios de desarrollo propios de la adultez. 
DE LA CONCEPCIÓN COMPENSATORIA A LA BASADA EN LAS CAPACIDADES DE LA EDUCACIÓN DE PERSONAS ADULTAS

\begin{tabular}{|c|c|}
\hline CONCEPCIÓN COMPENSATORIA & $\begin{array}{l}\text { CONCEPCIÓN BASADA EN } \\
\text { LAS CAPACIOADES }\end{array}$ \\
\hline $\begin{array}{l}\text { La educación básica debe finalizarse a la edad } \\
\text { adecuada, se convierte en una necesidad } \\
\text { compensatoria. }\end{array}$ & $\begin{array}{l}\text { La educación básica ha de adaptarse a la } \\
\text { presente evolución de la sociedad. }\end{array}$ \\
\hline $\begin{array}{l}\text { - Producto del pasado. } \\
\text { - Componente de subdesarrollo. } \\
\text { - La reforma como solución. } \\
\text { - Edad apropiada para aprender: preadultez. } \\
\text { - Paralelismo con el sistema escolar. }\end{array}$ & $\begin{array}{l}\text { - Producto de la evolución presente y futura. } \\
\text { - Componente esencial del desarrollo. } \\
\text { - Las reformas educativas producen el efecto } \\
\text { desnivelador. } \\
\text { - Cualquier edad es adecuada para aprender. } \\
\text { - Curriculum específico. }\end{array}$ \\
\hline
\end{tabular}

\section{Algunas propuestas educativas}

Transformando las escuelas en Comunidades de Aprendizaje

\section{Aprendizaje en la sociedad de la información}

En la sociedad de la información, el aprendizaje no depende tanto de lo que sucede en la clase, como de la correlación entre lo que pasa dentro del aula y lo que pasa en el resto de los sitios donde los niños y las niñas se desenvuelven en su vida diaria. Mientras todas las investigaciones más importantes concluyen con esta evidencia, el sistema escolar presenta una rigidez para transformarse y encajar en esta nueva demanda.

Las familias académicas ${ }^{3}$ hacen esta coordinación porque tienen en casa unos recursos educativos similares a los del aula lo que permite que estos niños aprendan más fácilmente que el resto. Las niñas y los niños que viven en estas familias encuentran los contextos sociales prioritarios, libros, ordenadores, conversaciones académicas y motivación hacia los conocimientos propios de la escuela en el mismo hogar. Además tienen más oportunidades para asistir a cursos de lenguas extranjeras en los países respectivos o para participar en actividades culturales privilegiadas.

Las familias no académicas tienen un contexto cultural diferente al de las escuelas. No tienen deficiencias culturales porque su contexto no es mejor ni peor, sino simplemente diferente. Pero esta diferencia se encuentra con una falta de coordinación y, consecuentemente, con el fracaso escolar y social. Como ya hemos visto previamente (el efecto desnivelador) más de la mitad de las familias están en esta situación. Los niños y niñas que forman parte de estos hogares son candidatos a ser socialmente excluidos en la sociedad de la información porque no tendrán las habilidades priori-

3. Llamamos familias académicas a aquellas que tienen al menos un miembro en activo con un título académico viviendo en la residencia familiar y que está diariamente interaccionando con los niños. 
zadas por ésta: la selección y el procesamiento de la información. En términos de Bernstein, tendrán códigos diferentes (Bernstein 1971, 1973, 1975).

Algunas alternativas situadas en el camino de la igualdad homogénea han tomado un modelo de educación remediadora atribuyendo déficits culturales a estos niños y niñas e intentando compensarlos con más recursos escolares. La atribución de déficits ha creado expectativas negativas. Por otro lado, los recursos adicionales son "más de los mismo" ya que no tienen en cuenta sus diferencias. Los niños y niñas de los sectores no privilegiados no se consideran diferentes sino menos motivados y con capacidades deficitarias, es decir, en términos de Apple, están interiorizando el efecto etiquetaje (Apple 1993).

Otras propuestas se sitúan en la orientación de la adaptación a la diversidad que parte del acercamiento de la escuela a la comunidad, infravalorando la dimensión instrumental del aprendizaje. Si los estudiantes no tienen un contexto tecnológico o académico, la escuela pone énfasis en otras dimensiones como los talleres artesanales o las habilidades que se relacionan con profesiones manuales. Estas alternativas han incrementado y han consolidado las desigualdades educativas y el fracaso escolar.

Las Comunidades de Aprendizaje son una alternativa en el camino de la igualdad de las diferencias. Se basan en el diálogo de toda la comunidad para construir y aplicar un proyecto que incluye las dimensiones comunicativas e instrumentales del aprendizaje y la promoción de las expectativas positivas. La relevancia del respeto a la diferencia y la solidaridad no se opone, sino que incluye el objetivo de aprender todas las habilidades de selección y procesamiento de la información requeridas para no estar excluido socialmente.

\section{El proceso de transformación}

Cuando empezamos a transformar un centro educativo en comunidad de aprendizaje, se inicia un profundo proceso. La primera fase es la formación del profesorado. Los puntos claves a tratar son la educación, el aprendizaje dialógico y la inteligencia cultural y qué representan en la actual sociedad de la información. Si los profesores están de acuerdo, es positivo que participen algunos miembros de la comunidad en algunos momentos de esta primera fase. Se trata de reflexionar sobre la realidad actual de los niños y de su futuro en la sociedad de la información. Por ejemplo, se compara la educación que ellos proporcionan a sus propios hijas e hijos y la razones que les han llevado a tomar dicha opción y la que ellos están dando a sus estudiantes. Después de esta formación, hay un periodo para reflexionar y madurar tras el cual el equipo tiene que llegar al consenso. Si un sector del profesorado no acepta el proyecto, su desarrollo no será posible.

Si el proyecto se lleva a la práctica, la comunidad se compromete a fomentar el aprendizaje de los estudiantes. Este proceso incluye la elaboración del proyecto de aprendizaje que la comunidad quiere, las tareas a llevar a cabo en los próximos dos años y la organización de los grupos deberá incluir la participación de profesionales de la educación y otras personas como voluntarios, parientes, instituciones y empresas. Cada comunidad desarrolla su propio proyecto pero hay algunos puntos en común: 
- El proceso de formación, limitado en la primera fase al profesorado, está abierta a todas las personas y a las instituciones que interaccionan con los niños y las niñas. Durante y después de la transformación, la formación en cuestiones de aprendizaje atañe a profesores, familiares, voluntarios, representantes de asociaciones y empresas. Asimismo, los recursos se consideran recursos para toda la comunidad: aulas de informática y bibliotecas que pueden ser utilizadas por los estudiantes, familiares y otros miembros de la comunidad.

- Porque la sociedad de la información es para todos, la educación en esta debe también ser para todos los estudiantes. El objetivo es superar el fracaso escolar y la exclusión social. La comunidad se moviliza para conseguir los recursos materiales y humanos que permitan desarrollar una perspectiva igualitaria. Uno de los eslógans es: En lugar de la cultura de la culpa, la cultura de la transformación. Si el Estado no nos proporciona ordenadores, los pediremos al Ayuntamiento y si no a las empresas. El objetivo no es justificarse argumentando que a la administración no le importan los socialmente excluidos, que las familias no están motivadas, etc. sino conseguir oportunidades reales para todo el mundo. Seguimos el mismo modelo con los recursos humanos: cooperamos con los voluntarios y las asociaciones para conseguir más horas y tener más personas adultas enseñando en el aula.

- Existe un pacto de consenso sobre el aprendizaje entre profesores, familiares y comunidad. Este pacto es el compromiso de cuidar entre todos del entorno educativo en todos los lugares donde los niños y las niñas desarrollan sus actividades. Por ejemplo, la biblioteca es una biblioteca con tutoría; los usuarios y usuarias se encontraran con diferentes personas adultas para orientarles en la lectura y no sólo para decirles qué libros hay.

\section{El origen del modelo}

La alternativa específica de las Comunidades de Aprendizaje se inicia en un centro de educación comunitaria en el barrio de La Verneda de Sant Martí de Barcelona, gestionado por las 1500 personas adultas que participan como estudiantes. Desde 1978 se ha comprobado que, en la sociedad de la información, un enfoque educativo adecuado contribuye a la superación de las desigualdades educativas y culturales en áreas de alto riesgo. El centro de La Verneda de Sant Martí, que está ubicado en una área periférica en Barcelona, ha estado trabajando en esta línea de superación desde sus inicios de una manera activa. Esto significa que todos los participantes y toda la comunidad se han comprometido activamente siguiendo el concepto de educación integrada, participativa y educación a lo largo de toda la vida. Algunos participantes que habían fracasado en la escuela primaria son actualmente personas con títulos universitarios o profesores de la universidad, directores de museos y directores de empresas.

Posteriormente, este enfoque se aplicó a escuelas infantiles y primarias y se aplicará en institutos de educación secundaria. Las tres orientaciones básicas, que se desarrollaran en las siguientes partes del artículo, son aprendizaje acelerado, aprendizaje dialógico e inteligencia cultural. Estas son las tres características principales de 
los proyectos educativos que están haciendo frente a los actuales cambios sociales en Europa.

\section{Aprendizaje Acelerado}

Antes de aplicar a las escuelas infantiles y de primaria el modelo del centro de educación comunitaria de La Verneda de Sant Martí, los miembros de CREA quisieron conocer otras experiencias similares a nivel internacional: las Escuelas Aceleradas, Escuelas de Éxito para todos y el Programa de Desarrollo Escolar. Aún con puntos similares a algunos de estos proyectos, nuestra opción fue aplicar el modelo de $\mathrm{La}$ Verneda centrándonos en un enfoque colectivo comunitario y no tanto en la aceleración del aprendizaje, aunque ésta sin una de sus características.

Por educación acelerada o aprendizaje acelerado entendemos todos los procesos que provocan un incremento significativo en el ritmo de aprendizaje en relación al que existía antes de la transformación. Normalmente, se ha aplicado a aquellos estudiantes que son considerados capaces de aprender más rápidamente que el grupo de referencia. Este objetivo puede ser fácilmente aplicable a procesos más rápidos (Unzicker 1932) que los habituales o situando a los estudiantes en clases más avanzadas que las que les correspondería por su edad (Klausmeier and Wiersma 1964). Con los superdotados, ha sido típico incrementar o elevar un nivel de sus estudios (Passow 1985).

Aunque estas dimensiones instrumentales o cognitivas han sido las que han dado más prestigio a esta perspectiva, contiene otros aspectos a destacar. Por ejemplo, es significativo que a menudo el concepto de educación acelerada se atribuye al movimiento creado por el psiquiatra búlgaro (Lozanov: 1978). También es remarcable que este autor llame a su propio movimiento sugestiología. En realidad, se basa en incrementar el aprendizaje de acuerdo con las habilidades que no se aprovechan normalmente, movilizándolas a través de la propuesta. Este aspecto está relacionado en gran parte con el incremento de expectativas positivas de los estudiantes.

Las experiencias aceleradas que están más conectadas con la perspectiva utilizada en los centros de educación de personas adultas como La Verneda de Sant Martí son las que se basan en niños y niñas desaventajados. A las dimensiones cognitivas y afectivas, estos últimos movimientos añaden un valor fundamental en educación de personas adultas: la igualdad. Estas alternativas emergen (Bereiter \& Engelmann 1966, Becker \& Carnine 1980) para superar la desigualdad entre estudiantes privilegiados y no privilegiados. Diferentes movimientos de educación acelerada están alcanzando un progreso importante tanto en el proceso de aprendizaje como en la solidaridad entre los estudiantes y sus familiares. 950 Escuelas Aceleradas, 50 Escuelas de Éxito para Todos, 160 que siguen el Programa de Desarrollo Escolar y otros proyectos que están probando que un enfoque educativo adecuado puede superar el fracaso escolar en las áreas de alto riesgo.

Las Escuelas Aceleradas se iniciaron en 1986 cuando Henry Levin, director de CERAS (Center of Educational Research at Stanford University) y sus colaboradores ponen en marcha un proyecto experimental para transformar dos escuelas de la bahía de San Francisco. Aproximadamente, el 80 por ciento de sus estudiantes vivían en áreas extremadamente pobres y tenían las peores notas del ránking estatal por su bajo 
nivel en matemáticas, lengua, etc. En pocos años, muchos de ellos pasaron a ocupar los primeros puestos como consecuencia del incremento del aprendizaje básico.

La clave está en la creencia de que todos los estudiantes son capaces de tener éxito y en el compromiso en la educación tanto de los profesores como de los familiares o de las diferentes organizaciones comunitarias. Son centros que aplican métodos y técnicas pedagógicas que tradicionalmente sólo se han utilizado en escuelas elitistas y por alumnos superdotados. El programa de Robert Slavin Éxito para todos empezó en septiembre de 1987 como resultado de la cooperación entre el Center For Research on Effective Schooling de la John Hopkins University y la Elementary School Abbottston de Baltimore. Todos los estudiantes del centro son afroamericanos y más de las tres cuartas partes de ellos están dentro de programas de comida gratuita por su precaria situación familiar. Robert Slavin y sus colaboradores prestaron una especial atención a los recursos académicos y sociales que han demostrado ser más eficaces para asegurar el éxito, aplicándolos y obteniendo unos resultados escolares fantásticos. La actividad se centra exclusivamente en el ciclo infantil y los niveles iniciales de educación primaria porque consideran los primeros años cruciales para garantizar el éxito.

El Programa de Desarrollo Escolar de James Comer empezó en 1968. Surgió como resultado de la cooperación entre Yale University Child Study Center y dos escuelas estatales en New Haven. Ambas escuelas tenían importantes dificultades. Las dos tenían el nivel más alto de fracaso escolar en el área y el nivel de absentismo era alarmantemente elevado. El Programa de Desarrollo Escolar es un proyecto de acción social que intenta ayudar a los estudiantes a través del cambio social. Se basa en un modelo psicoanalítico del desarrollo de los niños y niñas y en la psicología social. El programa propone acercar más la escuela y los hogares. Por esta razón, se requiere un trabajo conjunto entre los profesores, el personal de la escuela, los padres y los otros miembros de la comunidad para transformar el centro. En este sentido, la comunidad y la escuela tendrán más poder para luchar contra la pobreza y las condiciones de vida miserables.

\section{Inteligencia Cultural}

La segunda gran característica de los proyectos mencionados es la inteligencia cultural. Las Comunidades de Aprendizaje implican la participación de personas de cualquier edad y cultura en la comunidad para la promoción del aprendizaje de niños y niñas y adolescentes. Esta intervención necesita la confianza en la capacidad de aprender de todos ellos. El proceso de aprendizaje en la escuela tiene que estar orientado hacia la promoción de las habilidades y no hacia la compensación de supuestos déficits. Para conseguir este objetivo, los educadores no tenemos que partir de nuestros propios conocimientos, sino que tenemos que valorar positivamente el conocimiento y habilidades de los estudiantes. Esto, junto con los recursos didácticos más adecuados, conduce a una mejora significativa del proceso de aprendizaje y a terminar con las barreras.

Respecto a las personas adultas (padres, madres y otras familiares de los estudiantes), los estudios longitudinales (Schaie, 1983) prueban que la inteligencia puede incrementarse a lo largo de la vida. Por otro lado, las investigaciones sobre las dife- 
rencias entre inteligencia fluida y cristalizada (Cattel, 1971) y entre inteligencia práctica y académica (Scribner 1988) señalan que el aprendizaje puede basarse más en la práctica que en la académica. Si esto se hace al revés, los estudiantes se condenan al no aprendizaje o a un proceso lento.

La diferencia entre inteligencia fluida y cristalizada establecida por Raymond Cattell demuestra que mientras la primera (relacionada con el desarrollo psicológico) decrementa, la última (ligada a la experiencia) se mantiene y se incrementa. La inteligencia cristalizada da una respuesta específica (aunque sea limitada y provisional, lógicamente) a la pregunta de qué es la inteligencia. Además, hay que recordar la colonización de este concepto por lo establecido académicamente que nos lleva a no considerar como inteligencia medible todos aquellos procesos de resolución de situaciones que se han asimilado a lo largo de una vida integrada socioculturalmente.

Posteriormente, las tendencias en investigación han evolucionado hacia la inteligencia práctica, la cual se diferencia de la cristalizada porque se considera no sólo como una sola, sino como un amplio rango de inteligencias diferentes en diversos contextos sociales. Sylvia Scribner ha estudiado los procesos cognitivos y los contextos donde se desarrollan. Primero se basaron en una industria lechera, y después en el control informático de cantidades. Ceci y Liker (Ceci \& Liker 1986,1990) estudiaron otros aspectos de la inteligencia práctica. Concluyeron que la demanda real del mundo requiere el desarrollo de estilos y modos específicos que no solo son diferentes de los necesitados para el éxito académico sino que pueden ser opuestos a ellos. Ceci y Liker comprobaron que no había correlación entre las habilidades probadas y los resultados en Wechsler Adult Intelligence Scale. Además, descubrieron que una persona con un bajo nivel en el test es más compleja de analizar que otra con un nivel alto.

Sin embargo, todos las investigaciones han limitado su estudio a la dimensión cognitiva de aquellas relaciones humanas basadas en lo que Habermas denomina acción teleológica (Habermas: 1987). Dicha acción describe como las personas escogen sus objetivos y los medios para su consecución. Pero la interacción tiene más dimensiones; entre las diferentes acciones descritas por Habermas, la acción comunicativa está orientada hacia el entendimiento entre las personas. Cada educador sabe que la participación en el proceso educativo no sólo está orientada a conseguir unos determinados aprendizajes, sino que es y se espera que sea una relación con otras personas a través de la que también se adquieren conocimientos.

Actualmente se está desarrollando un concepto de inteligencia cultural basado en las experiencias prácticas en la escuela y en teorías como la de Habermas. La visión de la inteligencia cultural promueve un modelo de aprendizaje en el cual cada estudiante aporta su propia cultura. De este modo, los estudiantes consiguen una mayor confianza en sus habilidades e intensifican su proceso de aprendizaje desde un punto de vista cuantitativo y cualitativo. La educación basada en las teorías del déficit conlleva la creación de expectativas negativas respecto al aprendizaje como, por ejemplo, cuando se determina que una persona no sabe multiplicar porque no sabe hacerlo en la pizarra. Al contrario, el aprendizaje dialógico parte de la forma en que esta persona multiplica en su vida cotidiana, y de cómo es posible intensificar o mejorar su proceso de aprendizaje.

Por otra parte, es fundamental influir en las nuevas políticas desde este enfoque educativo. En muchas partes de Europa, existe el prejuicio de que las personas que no han terminado sus estudios primarios no son capaces de adaptarse a las nuevas tec- 
nologías. Esta exclusión se basa en su inhabilidad en leer o entender las instrucciones de las nuevas máquinas. Desde el aspecto comunicativo de la inteligencia cultural, se demuestra que estas personas son capaces de desarrollar nuevas tareas al ser formadas a través de sus habilidades comunicativas que consisten en la recepción oral de los contenidos.

\section{Del aprendizaje significativo al dialógico}

La característica más importante es el aprendizaje dialógico. Todas las investigaciones demuestran que el proceso de aprendizaje de niños y niñas depende más de la coordinación entre todas las actividades que desarrollan en los diferentes espacios de sus vidas (clase, hogar, calle) que no sólo de aquellos desarrollados en el aula. Sin embargo, hasta ahora las reformas educativas se han orientado hacia la intervención curricular por parte de los profesores, sin tener en cuenta a las familias y a las comunidades para participar en el proceso. Este error básico se basaba frecuentemente en una concepción obsoleta y mediocre del proceso de aprendizaje significativo. La perspectiva comunicativa propone un aprendizaje dialógico que incluye y supera el significativo. Para explicarlo, desarrollaremos tres concepciones: objetivista, constructivista y comunicativa.

Desde el objetivismo, una mesa es una mesa independientemente de si es utilizada por la gente o no; la realidad existe independientemente de los humanos. Basándose en Weber (1958/1904-5) y Husserl (1992), Schütz (1977/73) desarrolló una concepción constructivista, afirmando que la realidad social es una construcción humana que depende del significado que damos a nuestras propias acciones: una mesa es una mesa porqué la vemos como un objeto adecuado para actividades como escribir, jugar a cartas o comer. Habermas, entre otras personas y grupos, ha desarrollado una concepción comunicativa que incluye y supera el constructivismo de Schütz clarificando que los significados que damos a nuestras acciones dependen de todas las interacciones que tenemos con los otros. Una mesa es una mesa porque nos ponemos de acuerdo para utilizarla para escribir, jugar a cartas o comer.

Desde el objetivismo, la importancia de los contenidos es independiente de los estudiantes. Desde esta perspectiva los profesores deben poseer conocimientos sobre los contenidos que imparten y las habilidades metodológicas para enseñarlos (de aquí el dicho: sabe mucho pero no sabe enseñar). Los estudiantes tienen un nivel de comprensión diferente; algunas veces tratan de compensar los grupos menos aventajados con nuevos recursos didácticos. La formación del profesorado esta orientada a la asimilación de los contenidos y de la didáctica adecuada para transmitirlos. En su formación continua se pone énfasis en los aspectos didácticos porque el nivel de conocimiento de su formación inicial asegura el dominio de los contenidos, si son actualizados.

Desde el constructivismo, lo que es más importante no es la formación de los profesores sino el proceso de aprendizaje de los estudiantes. Desde esta posición, cada estudiante construye un significado diferente. Las habilidades para enseñar deben incluir la investigación de las diferentes maneras en que se construyen los significados y las formas de intervenir que las mejoran. Desde este enfoque, la formación de los profesores se orienta hacia el conocimiento de los procesos de aprendizaje de los 
individuos y de los procesos de construcción del conocimiento, siendo priorizados los aspectos psicológicos relacionados con la cognición.

La concepción dialógica incluye y supera la concepción constructivista, haciendo una importante precisión: el proceso de formación de los significados no sólo depende de los profesionales de la educación sino también de todas las personas y contextos relacionados con todos los procesos de aprendizaje de los estudiantes. Desde esta perspectiva, la formación del profesorado se orienta hacia el conocimiento de los individuos, los grupos de aprendizaje y hacia el conocimiento de cada materia desde un enfoque interdisciplinar. Este punto de vista incluye los aspectos pedagógicos, psicológicos, sociológicos y epistemológicos.

Los sistemas escolares que se basan en una concepción obsoleta del aprendizaje significativo elaboran sus planes independientemente de las familias y de la comunidad. En contraste, en las comunidades de aprendizaje hay un plan de cooperación en el proceso de aprendizaje en el que participan todos los grupos y personas que interactuan con los niños. En este sentido, los contratos de aprendizaje son firmados por los profesores, los familiares y las entidades especificando las condiciones para proseguir el proyecto y conseguir una educación de calidad para todos los estudiantes.

CARACTERÍSTICAS DEL APRENDIZAJE TRADICIONAL, CONSTRUCTIVISTA Y DIALÓGICO

\begin{tabular}{|c|c|c|c|}
\hline CONCEPCIÓN & OBJETIVISTA & CONSTRUCTIVISTA & COMUNICATIVA \\
\hline APRENDIZAJE & Enseñanza Tradicional & Enseñanza Significativa & Enseñanza Dialógica \\
\hline BASES & $\begin{array}{l}\text { La realidad es inde- } \\
\text { pendiente de los } \\
\text { individuos que la cono- } \\
\text { cen y la utilizan. }\end{array}$ & $\begin{array}{l}\text { La realidad es una } \\
\text { construcción social que } \\
\text { depende de los signi- } \\
\text { ficados que les dan las } \\
\text { personas. }\end{array}$ & $\begin{array}{l}\text { La realidad social es } \\
\text { una construcción } \\
\text { humana. Los signi- } \\
\text { ficados dependen de las } \\
\text { interacciones humanas. }\end{array}$ \\
\hline EJEMPLO & $\begin{array}{l}\text { La mesa es una mesa } \\
\text { independientemente de } \\
\text { como la ven las per- } \\
\text { sonas. }\end{array}$ & $\begin{array}{l}\text { La mesa es una mesa } \\
\text { porque nosotros la } \\
\text { vemos como un objeto } \\
\text { adecuado para comer. }\end{array}$ & $\begin{array}{l}\text { La mesa es una mesa } \\
\text { porque nos ponemos de } \\
\text { acuerdo para utilizarla } \\
\text { para comer. }\end{array}$ \\
\hline $\begin{array}{l}\text { CAPACIDADES } \\
\text { DE LOS } \\
\text { PROFESORES }\end{array}$ & $\begin{array}{l}\text { Contenidos a transmitir } \\
\text { y la didáctica para } \\
\text { hacerlo. }\end{array}$ & $\begin{array}{l}\text { Conocimiento del } \\
\text { proceso de aprendizaje } \\
\text { de los actores y de su } \\
\text { forma de construir los } \\
\text { significados. }\end{array}$ & $\begin{array}{l}\text { Conocimiento de los } \\
\text { procesos de apren- } \\
\text { dizaje de los individuos } \\
\text { y los grupos a través de } \\
\text { la construcción interac- } \\
\text { tiva de significados. }\end{array}$ \\
\hline $\begin{array}{l}\text { ENFOQUE } \\
\text { DISCIPLINAR }\end{array}$ & $\begin{array}{l}\text { Orientación pedagogica } \\
\text { que no tiene en cuenta } \\
\text { los aspectos psico- } \\
\text { lógicos, sociológicos } \\
\text { y epistemológicos. }\end{array}$ & $\begin{array}{l}\text { Orientación psicológica } \\
\text { que no tiene en cuenta } \\
\text { los aspectos peda- } \\
\text { gógicos, sociologicos y } \\
\text { epistemológicos. }\end{array}$ & $\begin{array}{l}\text { Orientación inter- } \\
\text { disciplinar: pedagógica, } \\
\text { psicológica, sociológica } \\
\text { y epistemologica. }\end{array}$ \\
\hline
\end{tabular}




\section{Bibliografía}

APPLE, M. 1993. Official Knowledge. Democratic education in a conservative world. London: Routledge.

BEQUER, W. C. \& CARNINE, D. 1980. Direct instruction: An effective approach for educational intervention with the disadvantaged and low permormers. Lahey, B.J. \& Kazdín, A. E. Advances in child clinical psychology, pp. 429-473. New York: Plenum.

BEREITER, C. \&ENGLEMANN, S. 1966. Teaching disadvantaged children in the preschol. Englewood Cliffs, NJ: Prentice-Hall, Inc.

BERNSTEIN, B. 1971. Class, Codes and Control. Vol. 1. Theoretical Studies towards a Sociology of Language. London: Routledge.

BERNSTEIN, B. 1973. Class, Codes and Control. Vol. 2. Applied Studies towards a Sociology of Language. London: Routledge.

BERNSTEIN, B. 1973. Class, Codes and Control. Vol. 3. Towards a Theory of Educational Transmissions. London: Routledge.

BLOOM, A. 1987. The Closing of the American Mind. How Higher Education Has Failed Democracy and Impoverished the Souls of Today's Students. New York: Simon and Schuster.

CARDOSO, F.: CAMOY, M.; CASTELLS, M. \& COHEN, S. 1993. The new economy in the information age. Reflections on our changing world. University Park: The Pennsylvania State University Press.

CASTELLS, M.; FLECHA, R.; FREIRE, P.; GIROUX, H.; MACEDO, D.; WILLIS, P. 1994. Nuevas perspectivas críticas en educación. Barcelona: Paidós.

CASTELLS, M. 1997. La era de la información. EConomía, Sociedad y Cultura. Madrid: Alianza Editorial.

CATTEL, R. B. 1971. The discovery of fluid and critallized general intelligence. Abilities: Their structure, growth and action. Boston: Houghton.

CECI, S. J. \& LIKER, J. 1986. Academic and Non academic intelligence: an Experimental Separation. Stemberg, R. J. \& Wagner, R. K. Practical Intelligence, p. 119-142. Cambridge, New York, New Rochelle, Melbourne and Sydney: Cambridge University Press.

$\mathrm{CECl}$, S. J. 1990, On intelligence... More or Less. A Bio-ecological Teatrise on Intellectural Development. Englehood Cliffs: Prentice Hall.

CORNER, J. P. 1980. School Power. Implications of an Intervention Project. New York: Simon \& Schuster.

CREA, 1997. Habilidades Comunicativas y desarrollo social. Investigación en proceso.

DELORS, J. 1996. La educación encierra un tesoro. Madrid: Santillana. Ediciones UNESCO.

Comisión de las Comunidades Europeas, 1995. Libro Blanco: Enseñar y aprender, hacia la sociedad cognitiva. Oficina de Publicaciones Oficiales de las Comunidades Europeas. Luxemburg.

FOUCALT, M. 1992. Genealogía del racismo. De la guerra de razas al racismo de Estado. madrid: La Piqueta.

FREIRE, M. 1992. A la sombra de este árbol. Barcelona: El Roure. 
GIDDENS, A. 1990. Structuration Theory and Sociological Analysis. Clark, J., Modgil, C. \& Modgil, S. 1990. Anthony Giddens. Consensus and Controversy. pp. 297 315. Londres: The Falmer Press.

HABERMAS, J. 1987. Teoría de la acción comunicativa. Vol l: Racionalidad de la acción y racionalización social. Vol Il: Crítica de la razón funcionalista. Madrid: Taurus.

HABERMAS, J, 1998. Ensayos políticos. Madrid: Península.

HABERMAS, J, 1998. La crisis del estado del bienestar y el agotamiento de las energías utópicas. Ensayos políticos. Madrid: Península, (Conferencia en el Parlamento Español el 26 Noviembre de 1984).

HABERMAS, J. 1989. Identidades nacionales y postnacionales. Madrid: Tecnos.

HABERMAS, J. 1996. Between facts and norms. Contributions to a Discourse Theory of Law and Democracy. Cambridge: Polity Press.

HABERMAS, J. 1997. Seminario The theory of democracy 9-10 de Abril. Barcelona: Universitat de Barcelona.

HIRSCH, E. D. 1987. Cultural Literacy. What Every American Needs to Know. New York: Vintage Books.

HOPFENBERG, W.; LEVIN, H.; MEISTER, G. \& ROGERS, J. 1990. Towards Accelerated Middle Schools fo At-Risk Students. Report of the Foundation Mc Connel Clark. Stanford, CA: Center for Educational Research at Stanford.

HUSSERL, L. 1992. La filosofía como ciencia estricta. Buenos Aires: Almagesto.

KLAUSMEIER, H. J. \& Wiersma, W. 1964. Effects on condensing content in mathematics and science in the junior and senior high school. School and Science Mathematics, v. 64, pp. 4-11. Green.

LEVIN, Henry M. 1972. "Shooling and inequality: The Social Science Objectivity Gap". Sadurday Review of Education. Vol LV. no 46, 11 de noviembre: 49-51.

LEVIN, Henry M. 1987. "New Schools for the Disadvantaged" a Teacher Education Quarterly, Vol 14, num. 4, pp. 60-83,

LEVIN, Henry M. 1989. "Financing the Education of At-Risk Students" a Educational Evaluation and Policy Anaysis. Vol. 11, num. 1, pp. 47-60. Washington.

LEVIN, Henry M. 1990. "At-Risk Students in a Yuppie Age" a Educational Policy. Vol. 4, num, 4, pp. 283-295.

LOZANOV, G. 1978. Suggestology and outlines of suggestopedy. New York: Gordon and Breach.

MARTIN, I., \& Sribner, S. 1991. Laboratory for cornitive studies of work: a case study of the intellectual implications of a new techonology. Teachers College Record. 582-602. New York: Teachers College. Columbia University.

MEC 1987. Proyecto de la Reforma Educativa. Una propuesta para debate. Madrid: Departamento de Educación y Ciencia.

MEC 1989a. Libro Blanco de la Reforma del sistema Educativo. Madrid: Departamento de Educación y Ciencia.

MEC 1989b. Diseño Curricular Base, Madrid: Departamento de Educación y Ciencia.

PASSOW H. 1985. Accelerated programs. The International Encyclopedia of Education: Research and Studies, v. 1, pp. 15-16. Oxford: Pergamon Press. 
POLFINGHOM, R.; Bartels, D. \& Levin, H. 1990. Accelerated Schools: The Inquiry Process and the Prospects of School Change. Trabajo presentado en la reunión anual de la American Educational Research Association. Boston, MA.

RORTY, R. 1989. Education Without Dogma. Truth, Freedom and Our Universities. Dissent. Primavera, pp. 1980204. New York: Foundation fo the Study of Independent Social Ideas.

SCHAIE, K. W. 1983. Longitudinal studies of adult psycological development. New York \& London: The Guyilford Press.

SCHUTZ, A. \& Luckmann, T. 1977. Las estructuras del mundo de la vida. Buenos Aires: Amorrortu.

SCRIBNER, S, 1988. Head and hand: An action approach to thinking. Teachers College, Columbia University. National Center on Education and Emplyment. (ERIC Document Reproduction Service No. CE 049 897), 16 pp.

SLAVIN, Robert E. \& Madden; Nancy, A. 1988. Instructionals Strategies for Accelerating the Achievement of Students At-Risk. Work presented to the "Conference on Acceleration of the Education of At-Risk Students". Stanford, CA. UNZICKER, S. P. 1932. A study of acceleration in the junior high school. The School Review, v. 40, pp. 346-356.

VIGOTSKY, L. S. 1986. Thought and language. Cambridge: MIT.

WEBER, M. 1958. The Protestant Ethic and the Spirit of Capitalism. New York, Charles Scribner's Sons. 
